\title{
Hypolipemiant Treatment: Making the Right Choice for Osteoporotic Patients
}

\author{
Șipoș RS1', Șuș loana², Pap Zsuzsanna1, Szalai Anna Szidónia1, Gabor Anamaria Victoria², Pávai Z1, \\ Brînzaniuc Klara ${ }^{1}$ \\ 1 Department of Anatomy and Embriology, University of Medicine and Pharmacy, Tîrgu Mureș, Romania \\ 2 Faculty of Medicine, University of Medicine and Pharmacy, Tîrgu Mureș, Romania
}

\begin{abstract}
Introduction: It has been shown that dyslipidemia is related to bone mineral density and fragility. Hypolipemiant drugs as statins or fibrates seem to increase the bone mineral density and probably to protect against fractures. The question that arises in this context is whether statins or fibrates have a positive effect on bone fracture repair process and which is their behaviour in an osteoporotic context. Our objective was to study the incidence of osteoporosis, dyslipidemia and of the association of these diseases, and to compare the effect of statins and fibrates on fracture repair in experimental conditions.

Material and method: We studied the incidence of dyslipidemia and osteoporosis in the activity of a private family medicine cabinet. In the experimental part we observed from a radiographic point of view the fracture repair process of rats' femurs. We analyzed 6 subgroups of 12 rats each: (1) ovariectomized control, (2) ovariectomized treated with statins, (3) ovariectomized treated with fibrates, (4) nonovariectomized control, (5) ) nonovariectomized treated with statins, (6) ) nonovariectomized treated with fibrates. The radiographic aspect has been objectified with a score at 2, 4, 6 and 8 weeks.

Results: From the total of 646 patients included in the study, 193 (29.87\%) had dyslipidemia while osteoporosis was diagnosed at 152 (23.53\%) patients. 301 (46.6\%) patients presented the association of these diseases. Comparing the subgroups of the OVX group, we had the following results: subgroup $1-5.5$ points, group $2-11$ points and group $3-4.5$ points. In the case of the NOVX subgroups, the scores were: subgroup $4-7.5$ points, subgroup $5-10$ points and subgroup $6-6.5$ points.

Conclusions: The fact that the incidence of dyslipidemia is higher than that of osteoporosis is an argument for the necessity of choosing a hypolipemiant treatment that has, at the same time, a protective effect on bone. Hypolipemiant treatment influences the fracture repair process. The positive effect of statins on this process is more important on the ovariectomized group, in contrast with fibrates which have an accentuated effect on the nonovariectomized group and this suggests an interference between hypolipemiant treatment and estrogens level. However, the treatment with fibrates delays the fracture repair, groups (3) and (6) scores being inferior to those of the control group. We sustain the helping effect of statins treatment on fracture repair process.
\end{abstract}

Keywords: dyslipidemia, osteoporosis, statins, fibrates, fracture

Received: 3 April 2011

\section{Introduction}

Osteoporosis is a disease represented by excessive loss of bone mass and a risk factor for fractures. According to WHO report for 2006, osteoporosis is an important problem of public health. 1700 fractures on osteoporotic bone happen daily (650 000 per year) in the European Union [1].

Osteoporosis and dyslipidemia are diseases associated with old age. Age, sedentary life style and estrogen deficiency are common risk factors for the two diseases. There are numerous reasons to search a proof that there is an association between bone mineral density and lipid profile [2]. Dyslipidemia has been associated with bone mass and fragility, and it could be a risk factor for osteoporosis [2-6], which suggests the importance of treating dyslipidemia in order to prevent osteoporosis and subsequent fractures.

In addition, therapeutic agents for osteoporosis have antiatherosclerotic properties, and molecules such as statins and fibrates, hypolipemiants, seem to increase bone mass

Correspondence to: Remus Sipos

E-mail: dr_rsipos@yahoo.com and even protect against fractures [7-11]. Experimental studies showed a positive effect of treatment with statins in the fracture repair process [12-15]. Regarding the effect of fibrates on fracture repair process, there are significantly less studies [11].

In this context the questions arise whether statins or fibrates have a comparable positive effect on fracture healing process and which is their behaviour in an osteoporotic context, so that we could be able to choose a treatment which could act in a polyvalent way, for the patient's benefit.

\section{Material and method}

The first part of the study contains a statistical analysis on the incidence of osteoporosis and dyslipidemia in a private family medicine practice, during the National Program for the Evaluation of Public Health.

The study comprises 646 patients investigated between 2007-2010. The analyzed parameters were: sex, age, osteoporosis, dyslipidemia, hepatopathy, antiosteoporotic and hypolipemiant treatment. The inclusion criteria were: presence of rheumatologically diagnostised osteoporosis or 
Table I. Animal groups

\begin{tabular}{cccccc}
\hline \multicolumn{1}{c}{ OVX } & \multicolumn{3}{c}{ NOVX } \\
\hline $\begin{array}{c}\text { Group } \\
\text { no. }\end{array}$ & Treatment & $\begin{array}{c}\text { Rats } \\
\text { no. }\end{array}$ & $\begin{array}{c}\text { Group } \\
\text { no. }\end{array}$ & Treatment & $\begin{array}{c}\text { Rats } \\
\text { no. }\end{array}$ \\
\hline 1 & $\varnothing$ (control) & 12 & 4 & $\varnothing$ (control) & 12 \\
2 & Simvastatin & 12 & 5 & Simvastatin & 12 \\
3 & Fenofibrate & 12 & 6 & Fenofibrate & 12 \\
\hline
\end{tabular}

dyslipidemia proven by total cholesterol level, with a reference value of $190 \mathrm{mg} / \mathrm{dl}$ and triglycerides level, with a reference value of $165 \mathrm{mg} / \mathrm{dl}$.

Due to the fact that in our clinical study the hypolipemiant treatment was diverse, which did not allow pertinent comparisons, we used an experimental model to evaluate and to compare the effect of the hypolipemiant medication on bone remodelling. For this purpose we chose the most frequently used active substances among our patients' medication, simvastatine and fenofibrate.

For the experiment part of the study we used $72 \mathrm{Al}-$ bino Wistar rats, all adult females, 16 to 18 months of age (human correspondent: women from 47 to 52 years of age, the period around menopause), weighing on average 300 grams. The Ethics Committee of the University of Medicine and Pharmacy of Tîrgu Mureș approved the treatment and study protocols with document number $2 / 2009$. The animals were divided into 2 groups: nonova-

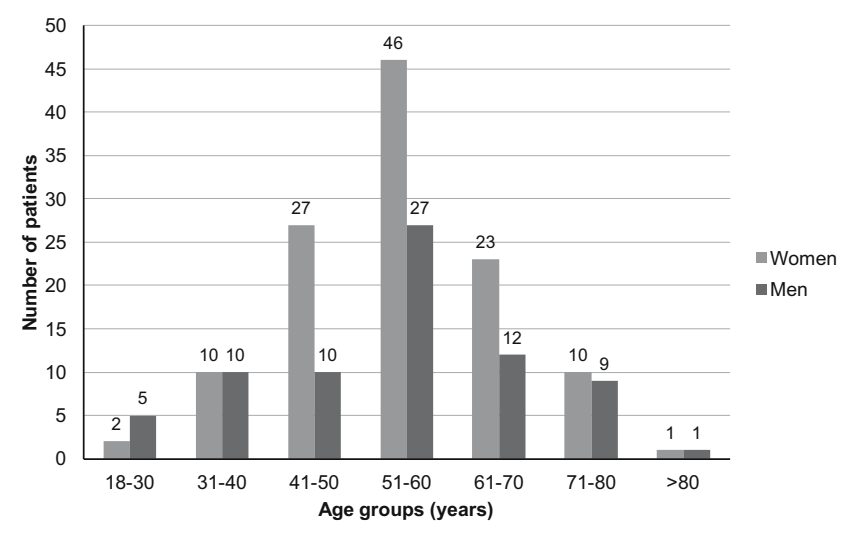

Fig. 1. Distribution of patients with dyslipidemia in age groups

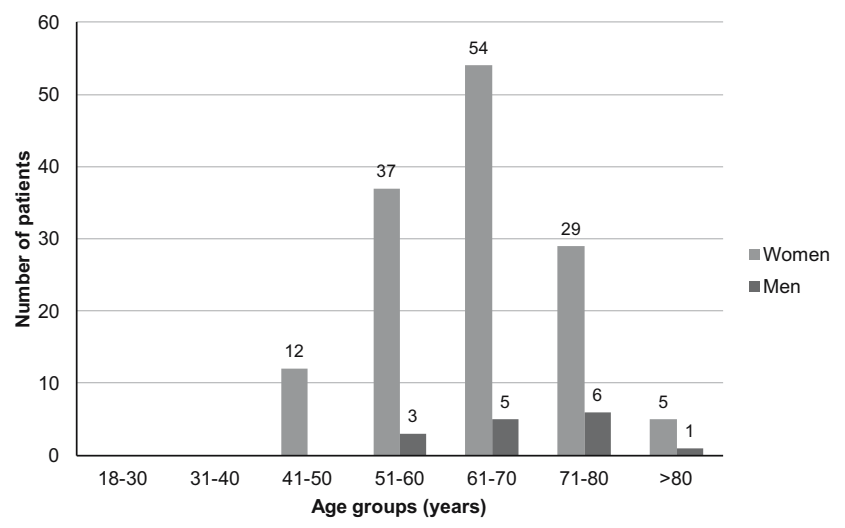

Fig. 3. Incidence of osteoporosis by age groups
Table II. Radiographic scoring system, adapted after Akman et al. [16]

\begin{tabular}{ll}
\hline Score & Modifications at the site of the fracture \\
\hline 0 & Lack of healing \\
1 & Callus formation \\
2 & Onset of bony union \\
3 & Disappearance of fracture line \\
4 & Complete bony union \\
\hline
\end{tabular}

riectomized rats (NOVX) and ovariectomized rats (OVX) (Table I).

For all surgical interventions the animals were anesthetized with an intraperitoneal injection of ketamine and xiline $(4-5 \mathrm{mg} / \mathrm{kg})$. All animals suffered a mediodiaphisary fracture to the right femur that was stabilized with an intramedullary rod. In the OVX groups the fracture was made at 12 weeks postovariectomy.

Groups 2 and 5 received a daily dose of simvastatin 10 $\mathrm{mg} / \mathrm{kg}$ and groups 3 and 6 received fenofibrate $10 \mathrm{mg} /$ kg. At 2, 4, 6 and 8 weeks postfracture radiographies were made in order to evaluate the healing process. To quantify the changes at the fracture site we used a radiographic score (Table II).

In order to evaluate the global effect on fracture healing process we used a personal algorithm: we made a ranking

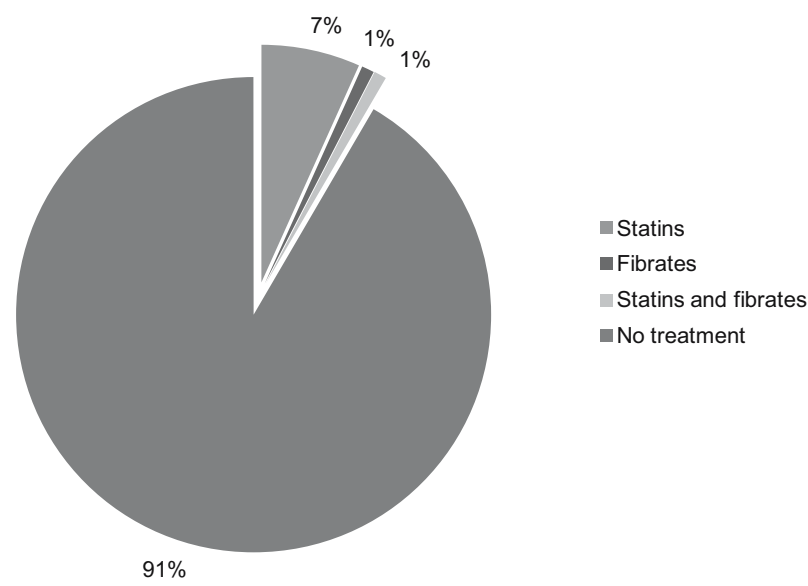

Fig. 2. Treatment of dyslipidemia for women

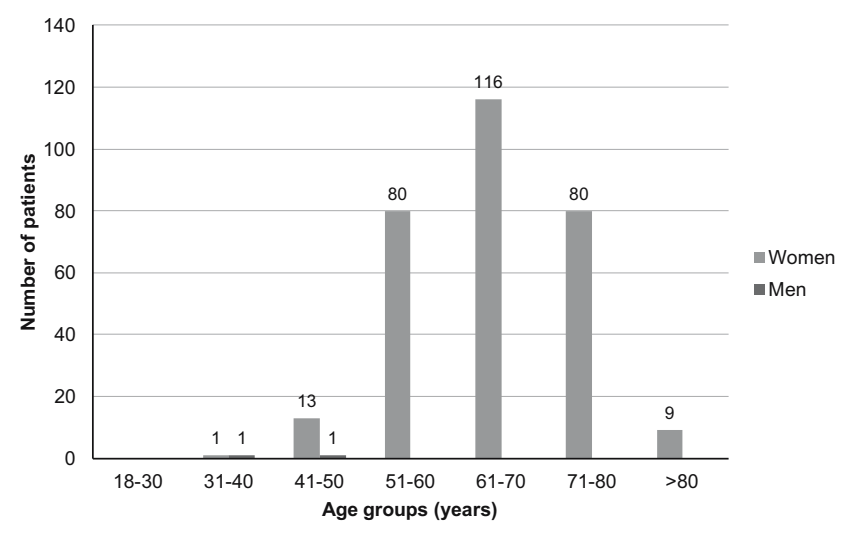

Fig. 4. Incidence of dyslipidemia and osteoporosis association 


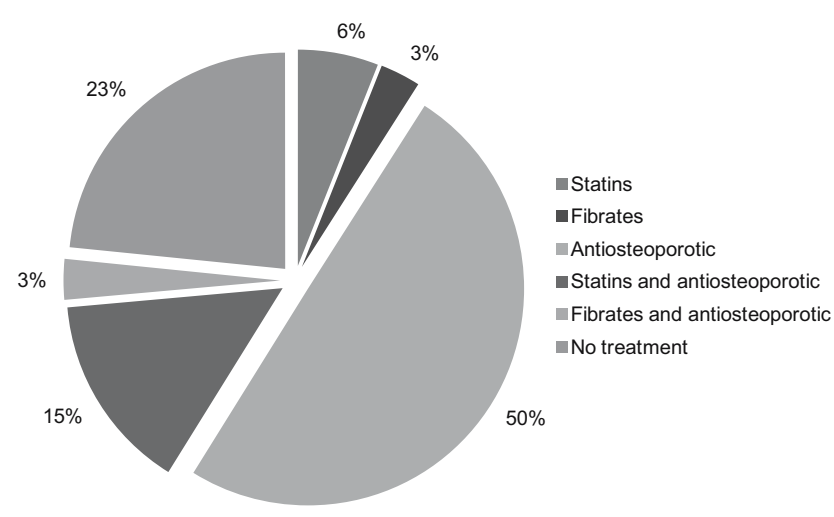

Fig. 5. Treatment for female patients with dyslipidemia and osteoporosis

between the subgroups belonging to each group at 2, 4, 6 and 8 weeks. The subgroup showing the most advanced stage of healing scored 3 points, the intermediate subgroup 2 points and the least evolved subgroup scored 1 point. In case of equality between two subgroups, each received the average of the unallocated scores (e.g. if a subgroup received 1 point and the other two groups had a similar aspect, each of them received 2.5 points).

\section{Results}

\section{A. Clinical results}

From the 646 patients included in our database, 555 were female $(85.91 \%)$. Osteoporosis alone was identified in 152 patients $(23.53 \%)$, dyslipidemia alone in 193 patients

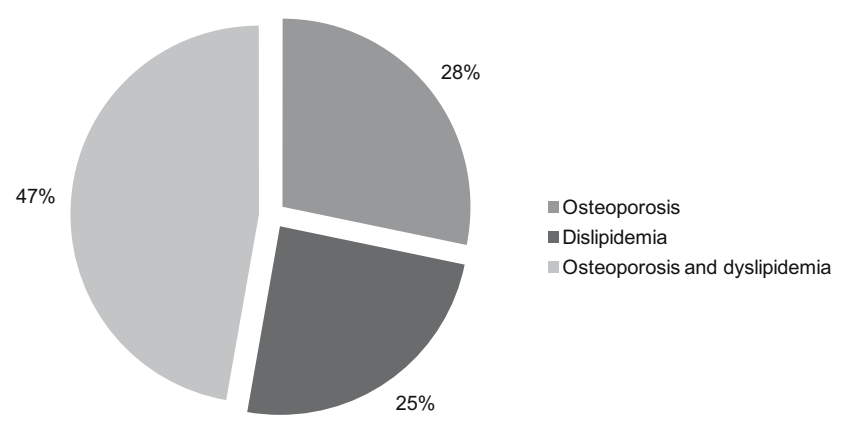

Fig. 6. Incidence of the two pathologies in women

(29.87\%) and the association between these two diseases in 301 patients $(46.60 \%)$.

Among the patients with dyslipidemia 61.65\% (119 patients) were women, representing $21.44 \%$ of all women included in this study. In the 51-60 years age group there were 46 female patients (38.66\% of women with dyslipidemia) (Figure 1).

Among female patients with dyslipidemia, 8 (6.72\%) were under treatment with statins, $1(0.84 \%)$ under treatment with fibrates, $1(0.84 \%)$ on statins and fibrates and $109(91.59 \%)$ had no hypolipemiant treatment (Figure 2).

In the group of patients with osteoporosis $90.13 \%$ (137 persons) were women, representing $24.68 \%$ of all women included in the study. The maximum incidence was in the sixth decade for women, 54 patients (39.42\%), and for men in the seventh decade, 6 cases (40\%)(Figure 3). Seventy-nine women $(57.66 \%)$ diagnosed with osteoporosis had an antiosteoporotic treatment.

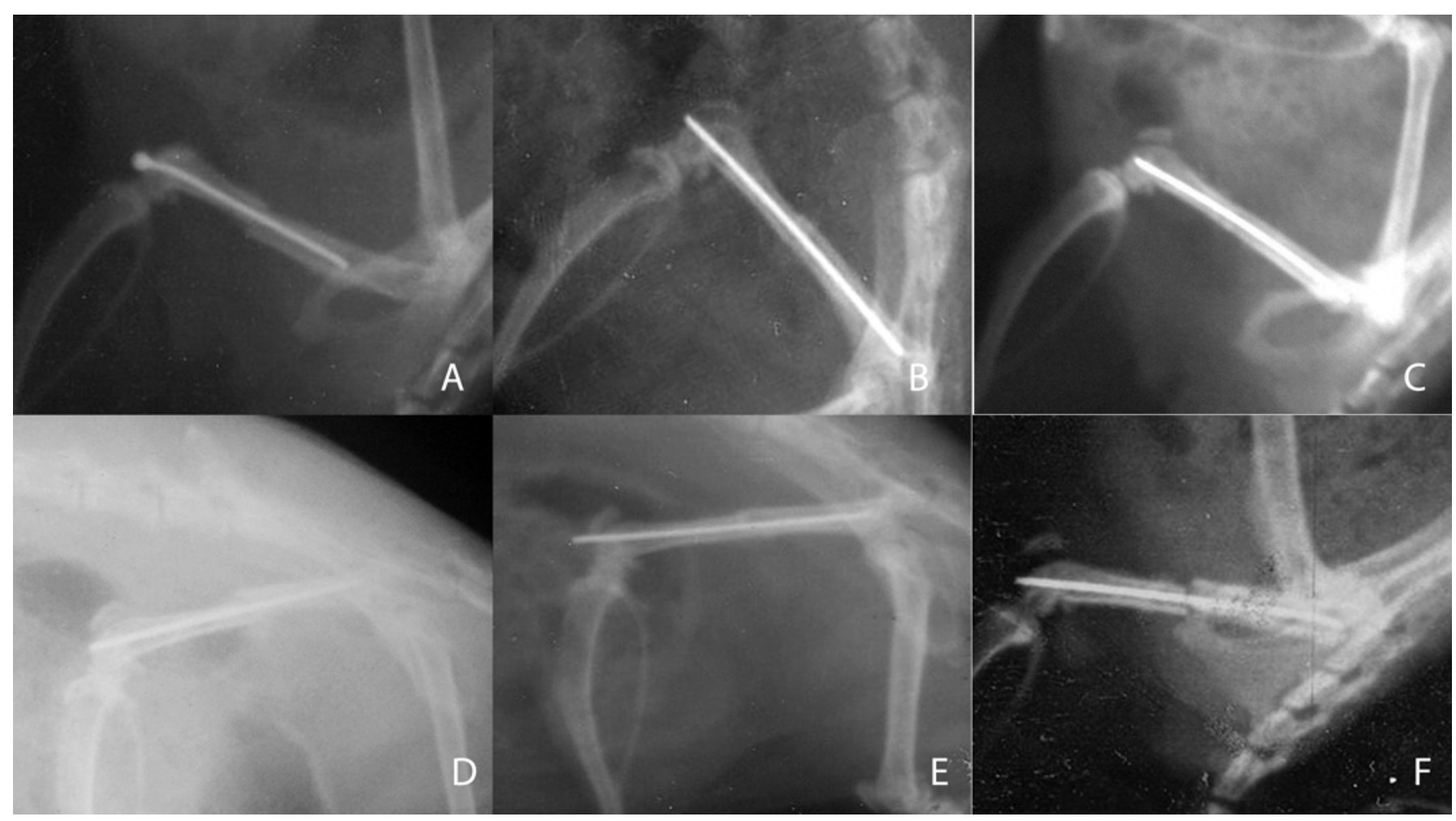

Fig. 7. Radiographic aspect at 2 weeks 


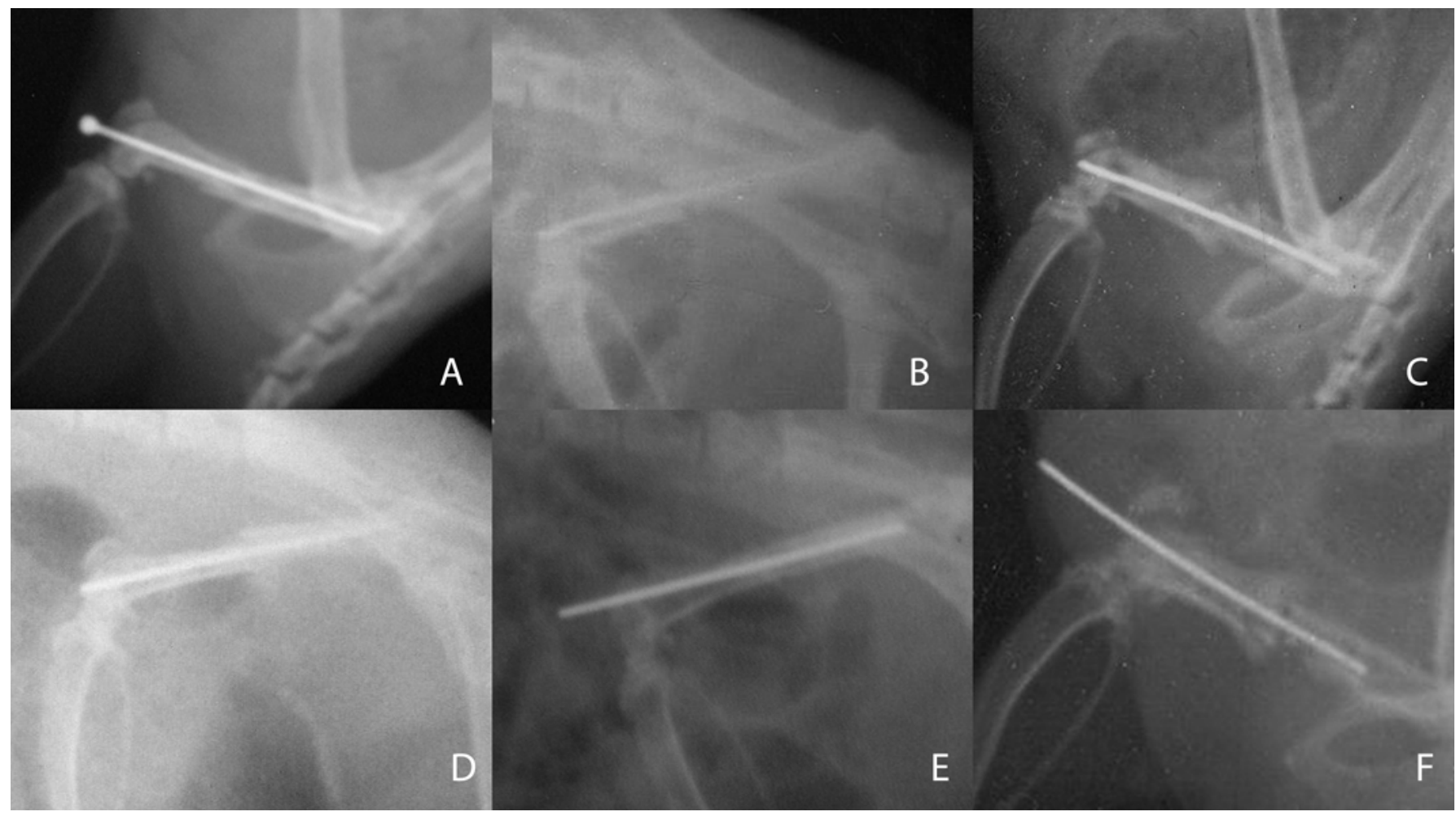

Fig. 8. Radiographic aspect at 4 weeks

The patients with dyslipidemia and osteoporosis were, in majority, represented by women, 299 (99.34\% of patients with both diseases). The maximum incidence of the association of the two diseases was in the sixth decade, 116 patients $(38.8 \%)$, followed by fifth and seventh decades with 80 patients each $(26.76 \%)$ (Figure 4$)$.
Female patients' treatment was as it follows: 149 $(49.83 \%)$ had only an antiosteoporotic treatment, 70 (23.41\%) had no treatment and 44 (14.72\%) had an associated treatment represented by statins and antiosteoporotic treatment (Figure 5).

Out of 418 women with dyslipidemia or dyslipidemia

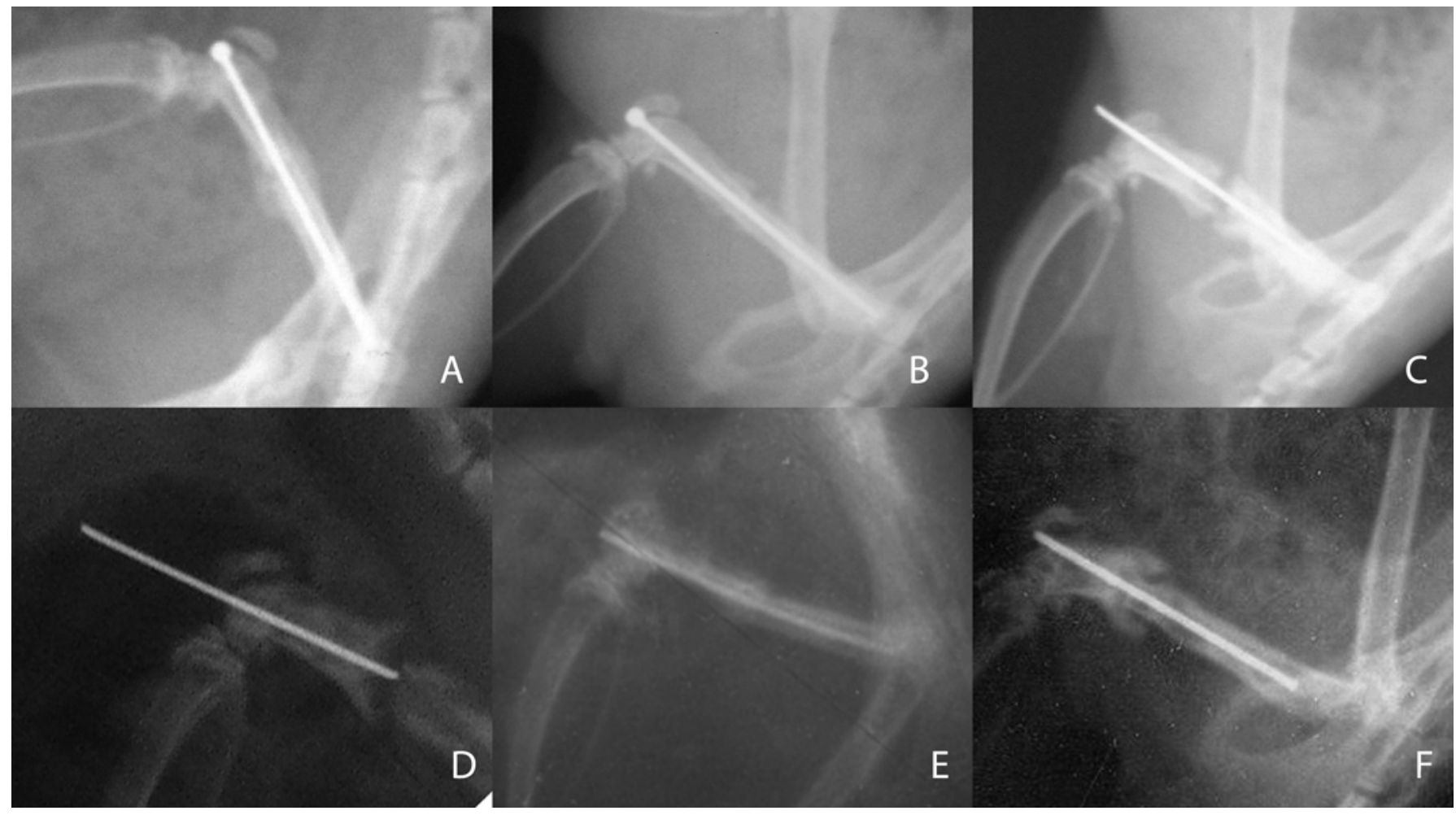

Fig. 9. Radiographic aspect at 6 weeks 


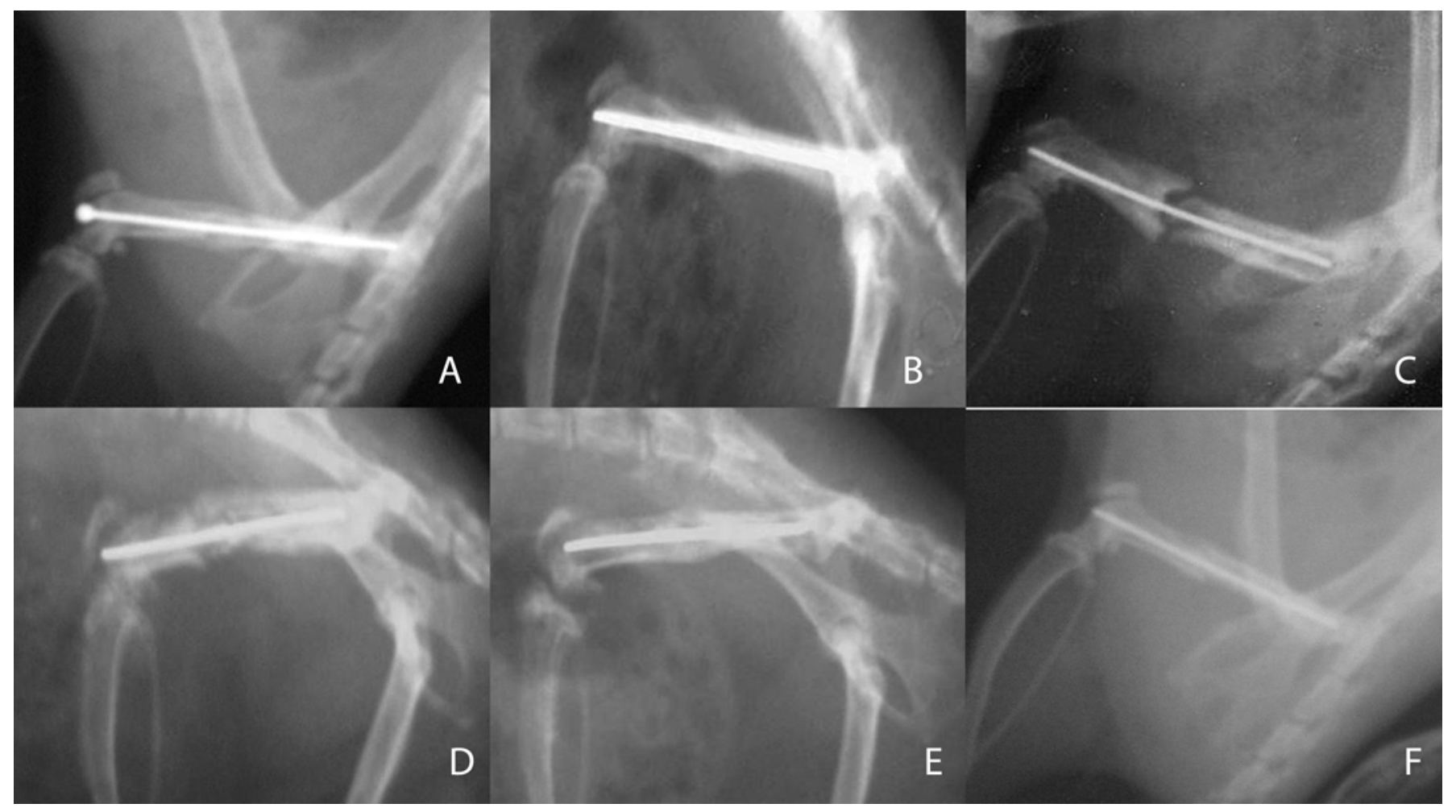

Fig. 10. Radiographic aspect at 8 weeks

associated with osteoporosis, 328 (78.47\%) had no hypolipemiant treatment.

\section{B. Experimental results}

Two weeks post fracture, the subgroups of NOVX group showed the same status of evolution of the healing process, respectively callus formation (Figure 7A,B,C). The same healing synchronisation was observed at 4 weeks (Figure 8A,B,C). But at 6 weeks, the NOVX subgroup treated with statins (Figure 9B) was completely consolidated, while NOVX subgroup treated with fibrates (Figure 9C) and NOVX control subgroup (Figure 9A) were still at the callus formation stage. The last evaluation at 8 weeks showed a complete healing in NOVX subgroup treated with statins (Figure 10B) the disappearance of fracture line in the NOVX control subgroup (Figure 10A) and the NOVX subgroup treated with fibrates (Figure 10C) was still in the formation of callus stage.

At two weeks the OVX subgroup treated with statins (Figure 7E) presented already the onset of bony union, the OVX control subgroup (Figure 7D) began the callus formation, while in the OVX subgroup treated with fibrates (Figure $7 \mathrm{~F}$ ) the callus formation process was not initiated. At

Table III. Radiographic scores

\begin{tabular}{lcccccc}
\hline & Group 1 & Group 2 & Group 3 & Group 4 & Group 5 & Group 6 \\
\hline 2 weeks & 1 & 2 & 0 & 2 & 2 & 2 \\
4 weeks & 2 & 2 & 0 & 2 & 2 & 2 \\
6 weeks & 2 & 2 & 1 & 2 & 4 & 2 \\
8 weeks & 2 & 4 & 2 & 3 & 4 & 2 \\
\hline
\end{tabular}

4 weeks, the radiographic aspect of the OVX control subgroup (Figure 8D) and of the OVX subgroup treated with statins (Figure $8 \mathrm{E}$ ) was similar, showing the onset of bony union, the OVX subgroup treated with fibrates (Figure 8F) did not begin the healing process. At 6 weeks, the OVX control subgroup (Figure 9D) and the OVX subgroup treated with statins (Figure 9E) has the same aspect as at 4 weeks, and the OVX subgroup treated with fibrates (Figure 9F) began the callus formation. Finally, at 8 weeks, the OVX subgroup treated with statins (Figure 10E) was completely healed, while the OVX subgroup treated with fibrates (Figure 10F) showed an onset of bony union, having a similar aspect with the OVX control subgroup (Figure10D).

Comparing the subgroups of the OVX group, we had the following results: subgroup $1-7$ points, group $2-11$ points and group $3-4.5$ points. In the case of the NOVX subgroups, the scores were: subgroup $4-7.5$ points, subgroup $5-10$ points and subgroup $6-6.5$ points.

\section{Discussions}

Osteoporosis and dyslipidemia are diseases that mainly affect the population aged more that 50 years old, leading to an increased morbidity and mortality. It is estimated that osteoporosis affects one of three women after menopause, especially after the age of 65 . In the population studied by us, $89.6 \%$ of women over 60 years old were affected by osteoporosis. In an EU report it is estimated that the number of women over 65 years old will increase to 26.4 million by 2050 [20].

A series of clinical studies examined the relation between dyslipidemia and bone mineral density (BMD) but 
the results were controversial [3-6, 17-19]. These observations added to the common risk factors for dyslipidemia and osteoporosis could be the explanation for the increased incidence of their association. In our study, the number of patients suffering of both diseases was greater than the number of patients having only one of them.

Considering the correlation between the two diseases and our results showing that dyslipidemia precedes osteoporosis (maximum incidence in the $5^{\text {th }}$ decade versus the $6^{\text {th }}$ decade) a hypothesis rises of a causal relation between the two pathologies. Knowing that women with an LDL-C higher than $160 \mathrm{ml} / \mathrm{dl}$ have a two-fold probability to suffer of osteopenia than women with a normal LDL-C [4] and that dyslipidemic patients, regardless of their gender, have an intensified bone turnover [5], new ideas for bone loss prevention and subsequent fractures appear.

After the identification of some molecular interferences between the two diseases, the behaviour of hypolipemiant treatment (with statins and fibrates) in an osteoporotic context has been experimentally evaluated. Some of these studies focused on the interference of these molecules with the fracture healing process, a major complication of osteoporosis. On a global level, a women's risk to suffer an osteoporotic fracture is of $40 \%$ [20].

Biphosphonates, the main treatment for osteoporosis, act a step below statins in the metabolic pathway of mevalonate [21]. Therefore, more and more evidence indicates the fact that these two therapeutic classes modulate at the same time osteoporosis and dyslipidemia. In experimental studies a better callus formation was showed in animals treated with statins in comparison to control group [12-15]. Our results are consistent with these studies, even though different means of evaluating fracture healing were used.

On the other hand, fibrates stimulate osteoblast differentiation and proliferation. By intensely stimulating ARNm for type I collagen, it leads to bone matrix formation. However, there is little information regarding treatment with fibrates in an osteoporotic context and in correlation with fractures [5].

Our experimental model show the beneficial effect that statins have on fracture healing formation on healthy and pathological bone (osteoporotic), the osteogenic effect being more precocious on osteoporotic bone. Fibrates play a role in inducing callus formation, their effect is favoured by a decreased estrogens level, but they delay the bone consolidation both on healthy and pathological bone.

\section{Conclusions}

The maximum incidence for osteoporosis, dyslipidemia and their association is superposable, that is the $5^{\text {th }}, 6^{\text {th }}$ and $7^{\text {th }}$ decades, with a peak in the $5^{\text {th }}$ decade for dyslipidemia and in the $6^{\text {th }}$ decade for osteoporosis and the association of the two diseases.

The higher incidence of the association between the two diseases, followed by the incidence of dyslipidemia in com- parison to the osteoporosis incidence brings arguments to the necessity of choosing a hypolipemiant treatment that has, at the same time, a protective effect on bone.

In the case of women, the incidence of these disease change their proportion, osteoporosis having a higher incidence than dyslipidemia not associated to osteoporosis. The percent of $78.46 \%$ represented by female patients with an associated pathology or only with dyslipidemia, with an exclusive antiosteoporotic treatment or without any treatment is the target group for optimizing the hypolipemiant treatment.

Hypolipemiant treatment influences the callus formation process. The effect of statins is more important on the OVX group, while for fibrates we can observe the opposite phenomenon, with a more accentuated effect on the NOVX group and that indicates the interference of hypolipemiant treatment with estrogens level. The treatment with fibrates delays callus formation, the scores of OVX subgroup treated with fibrates and of NOVX subgroup treated with fibrates being inferior to those of control subgroups.

Having an important incidence, osteoporosis needs the elaboration of treatment protocols for dyslipidemia that can not only decrease the cardiovascular risk but also the risk of complications of osteoporosis. We support the adjuvant effect of the treatment with statins in the fracture healing process, superior to the treatment with fibrates.

\section{References}

1. Johnell $O$, Hertzman P. What evidence is there for the prevention and screening of osteoporosis? WHO regional office for Europe 2006

2. Dennison EM, Synddall HE, Aihie Sayer A, Martin HJ, Cooper C. Lipid profile, obesity and bone mineral density: the Hertfordshire Cohort Study. Q J Med. 2007;100:297-303.

3. Yamaguchi T, Sugimoto T, Yano S, Yamaguchi M, Sowa $H$, Chen $Q$, Chihara K. Plasma lipids and osteoporosis in postmenopausal women. Endocrine Journal. 2002;49(2):211-217.

4. Poli A, Bruschi F, Cesana B, Rossi M, Paoletti R, Crosignani PG. Plasma low-density lipoprotein cholesterol and bone mass densitometry in postmenopausal women. Obstet Gynecol. 2003;102:922-926.

5. Majima T, Shimatsu A, Komatsu Y, Satoh N, Fukao A, Ninomiya K, Matsumura T, Nakao K. Increased bone turnover in patients with hypercholesterolemia. Endocrine Journal. 2008;55(1):143-151.

6. Saghafi H, Hossein-nezhad A, Rahmani M, Larijani B. Relationship between lipid profile and bone turnover in pre and postmenopausal women. Iranian J Publ Health. 2008;1:23-29.

7. Meier CR, Schlienger RG, Kraenzlin ME, Schlegel B, Jick H. HMG-CoA reductase inhibitors and the risk of fractures. JAMA. 2000;283(24):32053210.

8. Wang PS, Solomon DH, Mogun H, Avorn J. HMG - CoA reductase inhibitors and the risk of hip fractures in elderly patients. JAMA 2000, 283(24):3211-3216.

9. Wang JW, Xu SW, Yang DX, Lv RK. Locally applied simvastatin promotes fracture healing in ovariectomized rat. Osteoporos Int. 2007;18:16411650.

10. Chan A, Andrade SE, Boles M, Buist DSM, Chase GA, Donahue JG, Goodman MJ, Gurwitz JH, LaCroix AZ, Platt R. Inhibitors of hydroxymethylglutaryl-coenzyme $A$ reductase and risk of fracture among older women. Lancet. 2000;355(9222):2185-2188.

11. Syversen U, Stunes AK, Gustafsson BI, Obrant KJ, Nordsletten L, Berge $\mathrm{R}$, Thommesen L, Reseland JE. Different skeletal effects of the peroxisome proliferator activated receptor (PPAR) $\alpha$ agonist fenofibrate and the PPAR $\gamma$ agonist pioglitazone. BMC Endocrine Disorders. 2009;9:10-23.

12. Saraf SK, Singh A, Garbyal RS, Singh V. Effect of simvastatin on fracture healing - An experimental study. Indian J Experiment Biol. 2007;45:444449. 
13. Skoglund B, Aspenberg P. Locally applied Simvastatin improves fracture healing in mice. BMC Musculoskeletal Disorders. 2007;8:98.

14. Wang JW, Xu SW, Yang DS, Lv RK. Locally applied simvastatin promotes fracture healing in ovariectomized rat. Osteoporos Int. 2007;18:16411650.

15. Gutierrez GE, Edwards JR, Garret IR, Nyman JS, McCluskey B, Rossini G, Flores A, Neidre DB, Mundy GR. Transdermal lovastatin enhances fracture repair in rats. J Bone Miner Res. 2008;23:1722-1730.

16. Akman, Gogus A, Sener N, Bilgic B, Seckin BAF. Effect of diclofenac sodium on union of tibial fractures in rats. Advances in Therapy. 2002;19(3):119-125.

17. Zabaglia SF, Pedro AO, Pinto Neto Am, Guarisi T, Paiva LH, Lane E An exploratory study of the association between lipid profile and bone mineral density in menopausal women in a Campinas reference hospital. Cad Saude Publica. 1998;14(4):779-786.

18. Tanko LB, Bagger YZ, Nielsen SB, Christiansen C. Does serum cholesterol contribute to vertebral bone loss in postmenopausal women? Bone. 2003;32:8-14.

19. Solomon DH, Avorn J, Canning CF, Wang PS. Lipid levels and bone mineral density. The American Journal of Medicine. 2005;118:1414.e11414-e5.

20.Delmas PD, Fraser Mary. Strong bones in later life: luxury or necessity? Bulletin of the World Health. 1999;77(5):416-422.

21. McFarlane SI, Muniyappa R, Francisco R, Sowers JR. Pleiotropic effects of statins: lipid reduction and beyond. J Clin Endocrinol Metab. 2002;87:1451-1458. 